\title{
Two-hop Relay-based Vehicular Network in WiMAX
}

\author{
Nurul Nazirah M.I.M. \\ UTM-MIMOS CoE \\ Faculty of Electrical \\ Engineering \\ Universiti Teknologi Malaysia
}

\author{
Anis Izzati A.Z. \\ UTM-MIMOS CoE \\ Faculty of Electrical \\ Engineering \\ Universiti Teknologi Malaysia
}

\author{
Norsheila F. \\ UTM-MIMOS CoE \\ Faculty of Electrical \\ Engineering \\ Universiti Teknologi Malaysia
}

Sharifah Kamilah S.Y.
UTM-MIMOS CoE
Faculty of Electrical
Engineering
Universiti Teknologi Malaysia

\author{
Nurulashikin S. \\ UTM-MIMOS CoE \\ Faculty of Electrical \\ Engineering \\ Universiti Teknologi Malaysia
}

\author{
Mazlan A. \\ Wireless Communication \\ Cluster \\ MIMOS Berhad \\ Technology Park Malaysia
}

\begin{abstract}
In mobile communication, routing data through intermediate mobile node is a challenging task due to high mobility of nodes which causes rapid change in network topology and frequent disconnection between pair of nodes. To address these issues, Mobile Relay Station (MRS) is introduced into the network based on IEEE 802.16j standard. The MRS features are similar to the Fixed Relay Station (FRS), however the MRS has capability to move as Mobile Station (MS). In intermittent connection, by adapting the features of Delay Tolerant Network (DTN), the MRS can store and carry the data until it find the destination to forward the data. Assuming that MS is aware of its' own movement as well as its closest neighbor nodes. In this paper, Dynamic Routing Algorithm (DyRA) has been proposed to select next hop node towards the destination. The forwarding decision is based on a computed metric which is a combination of Packet Reception Rate (PRR), Link Expiration Time (LET) information and Number of Slots (NoS) available at the candidate MRS nodes. Also, the proposed Relay-based Vehicular Network (RVN) in WiMAX using cross layer design (CLD) is designed to select the optimal next hop node and set new route when current routing path is no longer available. The preliminary results for system throughput performance and average end-to-end delay are analyzed. The results show that by deploying relay-based in vehicular network can enhance system throughput in terms of packet loss ratio (PLR), packet delivery ratio (PDR), and average end-to-end (ETE) delay. NCTUns simulation environment has been used to study the mobile node performance.
\end{abstract}

\section{General Terms}

Routing, Vehicular network, WiMAX.

\section{Keywords}

Route selection, Link quality, Link stability, Cross layer design.

\section{INTRODUCTION}

The demand for wireless system in vehicular network has increased over the last several years. Vehicular network has the potential applications in providing support for Intelligent Transport System (ITS), multimedia and expediting the Internet access in highways [1]. Today, the technologies for establishing vehicular network include the IEEE 802.11b,
IEEE 802.11p and IEEE 802.16 standards. Taking advantage of longer communication range and higher data rate, IEEE $802.16 \mathrm{j}$ standard is proposed by standardization bodies to support mobile and multi-hop relay in vehicular network [2]. There are three types of Relay Station (RS) consist of fixed, nomadic, and mobile RS according to [3]. The fixed RS (FRS) is permanently set up at the same location. Even though the nomadic RS (NRS) is also fixed when operating, its position can be changed as needed. The last type of the RS is the mobile RS (MRS) which is moving in similar way as Mobile Station (MS).

Several routes between the MS and Base Station (BS) can be found when RSs are introduced into WiMAX networks. In mobile communication, routing data through intermediate mobile node is a challenging task due to high mobility of nodes which causes rapid change in network topology and frequent disconnection between pair of nodes [28]. Other issue in vehicular network is to decide which routing protocols could be the most suitable for data routing between MS and BS. To address these issues, Mobile Relay Station (MRS) is introduced into the network. The complications arise due to the fact that the MRSs are continuously moving. Thus, obtaining the end-to-end path can be a problem. Previous work in [4] assumes only the FRSs, not the MRSs.

Our work is focused on intermittent connection in Relaybased Vehicular Network (RVN). By adapting the features of Delay Tolerant Network (DTN), the MRS can store and carry the data until it find the BS to forward the data. In DTN, the fully connected path from source to destination is unfeasible to exist. This leads to a problem on how to route a packet from one node to another node in DTN. Most of the nodes in DTN are mobile, so that the connection is established when they enter into the transmission range of each other [29]. Assume that MS is aware of its' own movement, as well as its closest neighbor nodes sending their movements' information.

In this paper, Dynamic Routing Algorithm (DyRA) has been proposed to select next hop node towards the destination. The forwarding decision is based on the computed metric which is a combination of Packet Reception Rate (PRR), Link Expiration Time (LET) information, and Number of Slots (NoS) available at the candidate MRS nodes. The preliminary results for system throughput performance and average endto-end delay are analyzed. The results show that by deploying 
relay-based in vehicular network can enhance system throughput in terms of Packet Loss Ratio (PLR), Packet Delivery Ratio (PDR), and average end-to-end (ETE) delay.

The remaining part of this paper is structured as follows. Section II is the literatures that summarize other researchers work motivate to our work. Section III describes the overview of relay concept in IEEE 802.16j networks. Section IV deal with system description in vehicular network topology used for the proposed routing scheme. Section V is dedicated to our proposed routing scheme and parameters to be considered. Section VI describes the performance evaluation consist of simulation study and the results to analyze. Finally, our conclusions as well as future research directions are discussed in Section VII.

\section{RELATED WORK}

Research has been done in Mobile Ad Hoc Network regarding the communication between vehicles and access points using the traditional multi-hop routing technique called Ad-hoc Ondemand Distance Vector (AODV) [5]. AODV poses significant challenge including flooding and increasing control overhead in the network during route discovery process. However, the frequency of flooding can be reduced by extending the link duration between mobile nodes remain connected [6]. The decision is based on the computation of the stability factor called Link Expiration Time (LET).

To enhance the performance of AODV, the direction of moving node with respect to the next hop node is introduce in [7]. Node can identify the driving direction of other nodes by measuring the signal received from Doppler Effect [8]. Additional information about vehicle's movement is proposed in [9] combining of position, velocity, and direction to select next hop node towards the destination. The concept of link lifetime is also introduced to estimate the link duration between a pair of nodes remain connected.

When node density is high, multiples forwarder try to forward packets simultaneously cause broadcast storm problem in vehicular network [10]. An improved method of route discovery is implemented in [27] which proposed a protocol that select its neighbor node with some probability based on the network density, i.e. sparse or dense network region. To make multi-hop forwarding scheme more efficient, authors in [11] select the farthest node from the source node as the next hop node. Note that the distance between nodes affected link quality. The link quality is better when the distance between two nodes is small. Thus, the link with better channel condition offered high data rate, therefore more traffic can be sent at that time [12].

In opportunistic routing $[13,14]$, nodes require some information about their one-hop neighbors in order to decide on the next hop node. They frequently send out beacon messages announcing their current location, speed, and direction. However, high mobility rates in vehicular network can cause such information quickly becomes obsolete. Thus, more frequent beacon messages are required, increasing the overhead of the routing protocol.

Carry-and-forward scheme is the possible approach to overcome network disconnection problem. The idea of carryand-forward is adopted in $[15,16]$ where vehicle carry the packet until new vehicle enter the broadcast range and then forward the packet. However, authors in [17] agreed that this scheme has a drawback which may incur higher delay. Thus, they proposed an algorithm that selects an optimal route with least probability of network disconnection. But, this model only focuses on vehicle traffic in urban scenario influenced by traffic lights.

Several proposals highlighted on routing issues in relay-based WiMAX networks. In [18], the selection of paths through cross-layer approach is proposed considering the Signal-toNoise Ratio (SNR) from physical layer and buffer status from Medium Access Control (MAC) layer at the candidate RS to forward data from source to destination. Meanwhile, the authors in [19] proposed route selection scheme based on link quality and hop count. Only recently, some research on IEEE $802.16 \mathrm{j}$ for supporting mobile and multi-hop relay in vehicular communications has been conducted. To address the issues in vehicular network, the problem in selecting the optimal Relay Station (RS) in multi-hop relay network is studied in [20].

In this paper, we are trying to tackle route selection problem for Relay-based Vehicular Network (RVN) in WiMAX using cross layer design (CLD). Our aimed is to select the optimal next hop node which offer maximum throughput and set new route when current routing path is no longer available. The routing algorithm is expected to significantly improve throughput in RVN.The preliminary results show that two-hop communication outperforms one-hop communication as the traffic load increases in terms of PLR, PDR, and average ETE delay. Moreover, the concept of relay techniques in IEEE 802.16j standards is prominence to extend coverage area and supporting mobility in vehicular network. Table 1 exhibit the features of WiMAX network outperforms other networks in all aspect [21].

Table 1. Comparisons of wireless network technologies.

\begin{tabular}{|c|c|c|c|}
\hline $\begin{array}{c}\text { Wireless } \\
\text { Network }\end{array}$ & $\begin{array}{c}\text { ZigBee } \\
(\mathbf{8 0 2 . 1 5 . 4})\end{array}$ & $\begin{array}{c}\text { WiFi } \\
\mathbf{( 8 0 2 . 1 1} \\
\text { b/g/n) }\end{array}$ & $\begin{array}{c}\text { WiMAX } \\
(\mathbf{8 0 2 . 1 6} \text { e/j) }\end{array}$ \\
\hline Data Rate & $128 \mathrm{kbps}$ & $11 \mathrm{Mbps}$ & $30 \mathrm{Mbps}$ \\
\hline Range & $<150 \mathrm{~m}$ & $100-300 \mathrm{~m}$ & $3-5 \mathrm{~km}$ \\
\hline Mobility & LOW & LOW & HIGH \\
\hline
\end{tabular}

\section{OVERVIEW OF RELAY CONCEPT IN IEEE 802.16J}

The IEEE $802.16 \mathrm{j}$ is initialized to improve the performance of the IEEE 802.16e Mobile WiMAX standard via employment of relay stations (RS). RSs comes with the advantages of improving throughput at cell edges, extending coverage area of a base station (BS) and supporting high mobility for vehicular networks. There are two ways of forwarding data using RS, which are amplify-and-forward (AF) and decodeand-forward (DF). In AF scheme, RS amplify the signal received, and forward it to destination. As for DF scheme, the RS decodes the signal received before forwarding it to the destination. If there are no decoding errors, DF scheme always performs better with the cost of complexity and delay [30-32]. In this work, DF scheme is used.

As defined in the IEEE 802.16j Draft Amendment [2], relaying can be done in two modes: transparent mode and non-transparent mode. In transparent mode, the allowable number of hops is two as the RS only forwards the signal received without any preamble, DL/UL-MAP and DCD/UCD. In addition, this mode can only operate in centralized scheduling and RS employment is purposely for capacity enhancement. While non-transparent mode can support more than two hops communication as the RS transmits its own preamble, DL/UL-MAP and DCD/UCD messages. Thus, for 
this mode, not only does it enhanced user capacity but also increase coverage area of the BS. Both centralized and distributed scheduling can be used in this mode.

As for working in vehicular network, we focused on transparent mode relaying for one-hop and two-hop communication to study the behavior of mobile node. When dealing with multi-hop communication where nodes are moving with high speed, choosing the right path is crucial. The ability to select which direction the next hop is going to be should be the duty of MS.

\section{SYSTEM DESCRIPTION}

This section describes vehicular network topology used for the proposed routing scheme. We consider the scenario wherein the transparent network consists of one Base Station (BS) in a remote area, one or more Mobile Relay Stations (MRSs) and one mobile user node known as Mobile Stations (MSs). In this paper, we assumed that the MRSs communicate with the MS and BS only, and they do not communicate among themselves. Thus, all packets are forwarded between the MS and the BS involves two hops.

\subsection{Network Model}

In Relay-based Vehicular Network (RVN), one or more mobile RS (MRS) are allowed to be deployed between base station (BS) and mobile station (MS). As depicted in Fig 1, the BS is directly connected to the wired backhaul and provides connectivity management, and control of MRSs and MSs. The intermediate node (i.e. MRS) is responsible for relaying data between BS and MS. Appropriate selection of MRS to serve a particular MS is crucial in achieving good throughput. In highway, each vehicle is equipped with IEEE 802.16 technology represents as MS. The MS can be connected directly to BS or through MRS depending on the SNR value receives from super-ordinate station.
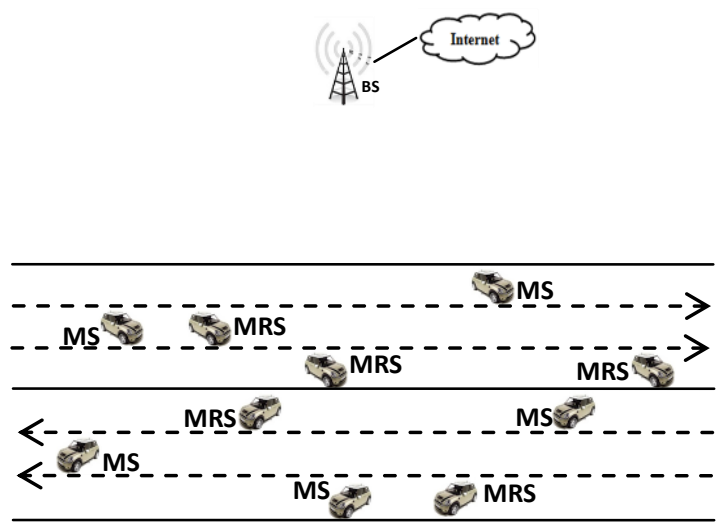

Fig 1: IEEE 802.16j vehicular networks.

The movement of MS is predicted by using mobility prediction proposed in [22]. MS is responsible to select MRS for data forwarding towards BS. In mobile communication, relay selection at one location may no longer help at another location. Thus, a dynamic relay selection scheme is necessary to improve the network performance in terms of throughput. A cross-layer design for Dynamic Routing Approach (DyRA) is proposed to overcome routing issues in vehicular networks.

As shown in Fig 2, MS closer to BS have the best SNR because they adopt the 64-QAM AMC coding scheme, but MS farther from BS have the worst SNR because BPSK is used instead. As a result, MS selects the RS with the best SNR with the highest AMC coding rate to determine path in multihop relay networks.

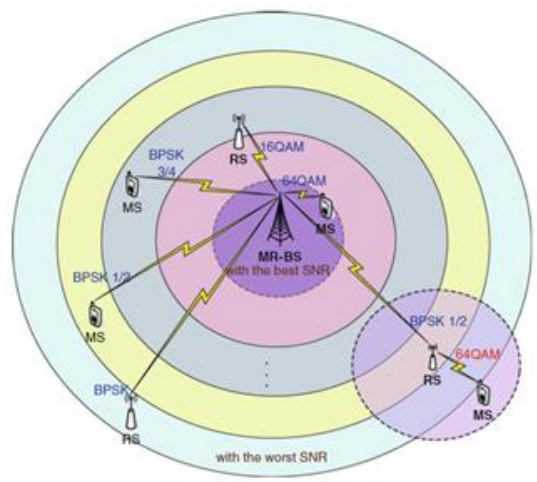

Fig 2: Different Adaptive Modulation and Coding (AMC) coding rates under various SNR.

Since we focused on transparent mode relaying for two-hop communication, as stated in NCTUns-6.0, the path selection is based on the weightage of SNR received at BS, RS and MS and is calculated by the BS using centralized scheduling. The SNR reflected the condition of wireless link and is used to specify the required modulation and coding rate for each transmitted packet on a link. Table 2 shows the relationship between SNR, modulation type and coding rate [23]. The BS decides the MS's access station according to the weight of the link between MS and RS, and the weight of the link between RS and BS. The weight of a link corresponds to the used modulation and coding rate on that link. The BS will choose to use the RS as the MS's access station if the following condition is met,

$$
W_{r}+W_{p}<W_{s}
$$

where,

- $W_{s}=$ the corresponding weight of BS-MS.

- $W_{r}=$ the corresponding weight of MS-RS.

- $W_{p}=$ the corresponding weight of RS-BS.

Table 2. Modulation and Coding Scheme (MCS) table.

\begin{tabular}{|c|c|c|c|c|c|}
\hline $\begin{array}{c}\text { Receiv } \\
\text { ed } \\
\text { SNR } \\
\text { (dB) }\end{array}$ & $\begin{array}{c}\text { Modulati } \\
\text { on }\end{array}$ & $\begin{array}{c}\text { Codi } \\
\text { ng } \\
\text { Rate }\end{array}$ & $\begin{array}{c}\text { Modulat } \\
\text { ed } \\
\text { Symbol }\end{array}$ & $\begin{array}{c}\text { Bits } \\
\text { per } \\
\text { symb } \\
\text { ol }\end{array}$ & $\begin{array}{c}\text { Weight } \\
\mathbf{W} \\
\text { (Symb } \\
\text { ols per } \\
\text { bit) }\end{array}$ \\
\hline 5.0 & QPSK & $1 / 2$ & 2 & 1 & 1 \\
\hline 8.0 & QPSK & $3 / 4$ & 2 & $3 / 2$ & $2 / 3$ \\
\hline 10.5 & $16-\mathrm{QAM}$ & $1 / 2$ & 4 & 2 & $1 / 2$ \\
\hline 14.0 & $16-\mathrm{QAM}$ & $3 / 4$ & 4 & 3 & $1 / 3$ \\
\hline 16.0 & $64-\mathrm{QAM}$ & $1 / 2$ & 6 & 3 & $1 / 3$ \\
\hline 18.0 & 64-QAM & $2 / 3$ & 6 & 4 & $1 / 4$ \\
\hline 20.0 & 64-QAM & $3 / 4$ & 6 & $9 / 2$ & $2 / 9$ \\
\hline
\end{tabular}

Once the modulation type and coding rate is chosen, the data rate of link can be obtained as follows [24],

$$
\text { DataRateofLink }=\frac{N_{\text {OFDMA }} * N_{S u b c} * C R * \log _{2}(\mathrm{mod})}{F_{d}}
$$

where,

- $\quad N_{O F D M A}=$ the number of OFDMA symbols per slots. 
- $\quad N_{s u b c}=$ the number of subcarriers per slot.

- $\quad C R=$ the coding rate.

- $\bmod =$ the modulated symbol.

- $F_{d}=$ the frame duration.

In this paper, we considered a transparent mode. Thus, it is assumed that the ranging procedure is successfully done at the beginning.

\subsection{Ranging Procedure}

There are two types of RS defined in IEEE802.16j which includes transparent RS and non-transparent RS. In MMR network with RS working in transparent mode, a MS is synchronized with MRBS and received MAPs from it since transparent RS does not transmit preamble and MAPs. Therefore, it does not recognize the existence of RS even though the MS communicate with the MRBS through transparent RS. Besides, only centralized scheduling mode is allowed to be employed in this mode. In contrary to transparent RS, the RS working in non-transparent mode transmit preamble and MAPs to their subordinate stations. Thus, it is possible for non-transparent mode to apply centralized or distributed scheduling mode.

When a new node requests to enter the network, it shall do the ranging procedure first. Ranging for both RS type follows different task. The details on ranging procedure are explained in [2].

\section{PROPOSED WORK}

This section describes several assumptions to be made for supporting the proposed routing scheme and some parameters we deal with include the physical and MAC layer parameters in order to make an optimal routing decision. The idea of link lifetime is also applies to check the validity of the selected neighbor node.

\subsection{Assumptions}

There are several assumptions to be made to cope with the proposed network topology as depicted in Fig 1:-

- All mobile nodes, i.e. MS and MRS on the road participate in sending and relaying data packets whereas the market penetration of wireless-equipped mobile nodes is 100 percent.

- No sudden changes of direction while it is traveling. Overtaking maneuver is out of concern in this paper.

- Each node's trajectory follows a straight line.

- Constant velocity for all nodes, i.e. the speed is between $616.00 \mathrm{kmph}$ to $120 \mathrm{kmph}$. Mobile node cannot accelerate and decelerate.

\subsection{Dynamic Routing Algorithm (DyRA)}

Vehicular network suffer from random variation in channel and network condition. This leads to poor performance in RVN especially with a conventional non-flexible routing. To cope with this instability, a cross-layer design that supports dynamic changing parameter values across multiple layers of protocol stack is needed. In this paper, we considered the Signal-to-Noise Ratio (SNR), Packet Reception Rate (PRR) and Link Expiration Time (LET) from physical layer and Number of Slots (NoS) available from Medium Access Control (MAC) layer at the candidate MRS for route decision at network layer as shown in Fig 3.

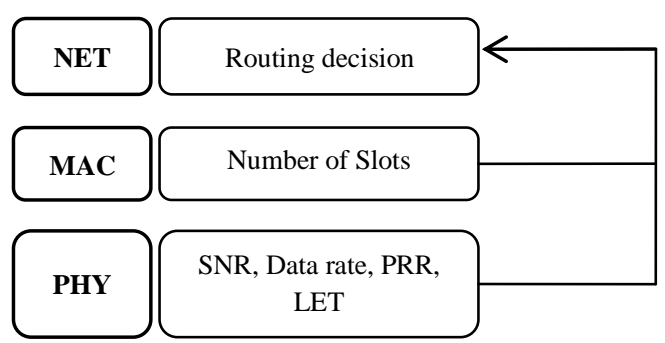

Fig 3: Cross-layer design (CLD).

The concept of link lifetime is used as an attempt to improve network performance in vehicular network. The link lifetime is defined as the duration of time for nodes remains connected provided that such nodes are within the radio range of each others. By using the movement parameters, i.e. position and speed of two neighbor nodes, we can check the validity of the next hop nodes candidate.

As shown in Fig 4, suppose that MS1 moves with speed of 90 kmph and wants to send packet to BS. It first broadcasts a query searching for a neighboring node which can reach BS. There are four candidates node within MS1 coverage, i.e. MRS1 (75 kmph), MRS2 (110 kmph), MRS3 (115 kmph), and MRS4 (80 kmph). By considering certain parameters, MS1 will select the optimal route for sending data packet to destination.
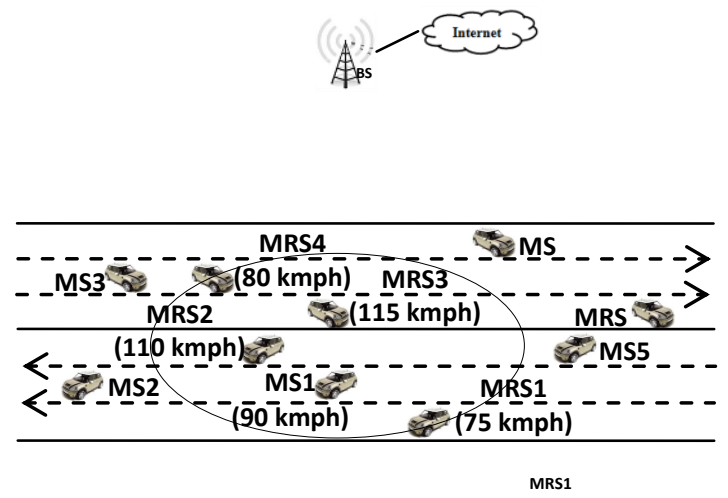

Fig 4: Neighbor discovery - MS node broadcast BEACON_CTRL_MSG.

The proposed DyRA performed route selection based on link quality (i.e. PRR), link stability (i.e. LET) between two nodes remain connected and Number of Slots (i.e. NoS) information from the candidate MRS node. In finding available path, we introduced new signaling message called BEACON_CTRL_MSG for neighbor discovery. This message is broadcast by MS and one hop neighbor of MS, i.e. MRS will receive this message and reply back with its movement information. As depicted in Fig 5, BEACON_CTRL_MSG contains field of Node ID, speed, position, direction, and timestamp. This signaling message is useful to check the validity of the link between nodes are remain valid before sending data packet. Upon receiving BEACON_CTRL_MSG, first MS checked either it has received this message previously or not. If yes, MS will drop this message immediately. However, if not, MS will proceed with updating the cost function. 


\begin{tabular}{|c|c|c|c|c|}
\hline \multicolumn{5}{|c|}{ BEACON_CTRL_MSG } \\
\hline $\begin{array}{c}\text { Node } \\
\text { ID }\end{array}$ & Speed & Position & Direction & $\begin{array}{c}\text { Timestamp } \\
\text { (Current time) }\end{array}$ \\
\hline
\end{tabular}

Fig 5: Packet format for BEACON_CTRL_MSG.

Therefore, if the motion parameters of two nodes are known, we can determined the duration of time these two nodes will remain connected. Let consider two nodes, $i$ and $j$ are within the transmission range $r$ of each other, speed $v_{i}$ and $v_{j}$, coordinates of mobile node $i,\left(x_{i}, y_{i}\right)$ and $\left(x_{j}, y_{j}\right)$ be that of mobile node $j$, and movement direction angles $\theta_{i}$ and $\theta_{j}$, respectively. Then, the link lifetime called LET is predicted by [22],

$$
L E T=\frac{-(a b+c d)+\sqrt{\left(a^{2}+c^{2}\right) r^{2}-(a d-b c)^{2}}}{a^{2}+c^{2}}
$$

where,

- $\quad a=v_{i} \cos \theta_{i}-v_{j} \cos \theta_{j}$

- $b=x_{i}-x_{j}$

- $\quad c=v_{i} \sin \theta_{i}-v_{j} \sin \theta_{j}$

- $\quad d=y_{i}-y_{j}$

Note that when $v_{i}=v_{j}$ and $\theta_{i}=\theta_{j}$, LET becomes infinity, $\infty$. By predicting the LET, only neighbor nodes that are still within the transmission range will be considered as the valid candidates for the next hop selection.

As discussed in [25], we obtained the expected probability of successfully receiving a message at distance $d$ while considering a communication range of $r$ in meter as follows,

$$
\operatorname{PRR}(d, r)=e^{-3\left(\frac{d}{r}\right)^{2}}\left(1+3\left(\frac{d}{r}\right)^{2}+\frac{9}{2}\left(\frac{d}{r}\right)^{4}\right)
$$

We assume that the transmission range $r=500 \mathrm{~m}$ of each node except BS. Note that the distance $d$ is compute from SNR received from super-ordinate node. Therefore, we are able to calculate PRR by assuming the noise level to be constant over the geographical space. However, by predicting only PRR is not guaranteed the link stability of mobile nodes link. We improve the stability by estimating the LET of link between two adjacent mobile nodes. Based on this prediction, the selection is not just based on link quality but also link stability.

We also measure the load by looking at the Number of Slots (NoS) available at the MRS candidates nodes. As the MS want to send data, we can calculate how many slot requires [24]. Thus, a packet with size $p$ bytes requires a total number of slots given by,

$$
\text { NumberofSlots }=\operatorname{ceil}\left(\frac{8 p}{\text { BitsperSlot }}\right)
$$

It was shown in [26] that the optimal packet size in IEEE 802.16 is limited to power of two values in the range of 16 to 1024 bytes. The packet size is limited to this range and for each transmission the packet size is $2^{N}$ bytes where $N$ is from 4 to 10 . The number of bits per slot can be computed as follows,

$$
\text { BitsperSlot }=N_{O F D M A} * N_{\text {Subc }} * C R * \log _{2}(\bmod )
$$
where,

- $\quad N_{O F D M A}=$ the number of OFDMA symbols per slots.

- $\quad N_{s u b c}=$ the number of subcarriers per slot.

- $\quad C R=$ the coding rate

- $\bmod =$ the modulated symbol.

The theoretical throughput can be calculated by using the following equation,

$$
\text { Throughput }=\frac{\text { BitsperSlot } * \text { NumberofSlots }}{F_{d}}
$$

DyRA is designed to match the requirements of vehicular network. The core idea of DyRA is to define a function while selecting the next hop node. The route cost function is called Cost Function $(C F)$ depends on three factors, i.e. PRR, LET and NoS available at the candidate MRS node. The $C F$ computed by MS at the current time is defined as follows,

$$
\begin{aligned}
C F_{M S-M R S i} & =\alpha L E T_{M S-M R S i}+\beta P R R_{M S-M R S i} \\
& +\gamma N O S_{M R S i}
\end{aligned}
$$

where, $\alpha+\beta+\gamma=1$.

Thus, a sorted list of next hop nodes candidate can be defined based on the $C F$. The optimal route can be determined by,

$$
O R=\max \left(C F_{1}, C F_{2}, \ldots, C F_{n}\right)
$$

where, $n$ is the number of available route. The node with the highest route cost among all the candidate nodes will be chosen as the optimal next hop node.

Note that in the route selection phase, the selection of optimal route can be depends on criterion such as route Quality of Service (QoS), traffic load, etc. In this paper we only studied the preliminary results on system throughput and average ETE delay between one-hop and two-hop communication. For the sake of simplicity, the simulation is done from the simplest network topology consists of one source node, one destination node and a relay node shown in Fig. 6. In future work, we will look at other factors in DyRA for optimal route selection in relay-based vehicular network environment.

\section{PERFORMANCE EVALUATION}

This section describes WiMAX network topology operating in transparent mode. The result for system throughput according to traffic load condition and average end-to-end delay is also discussed.

\subsection{Simulation Study}

Simulation is done using NCTUns. The network consists of Base Station (BS) connected to Relay Stations (RSs), and Mobile Stations (MS) constructed using IEEE 802.16j transparent mode topology as shown in Fig 6. 


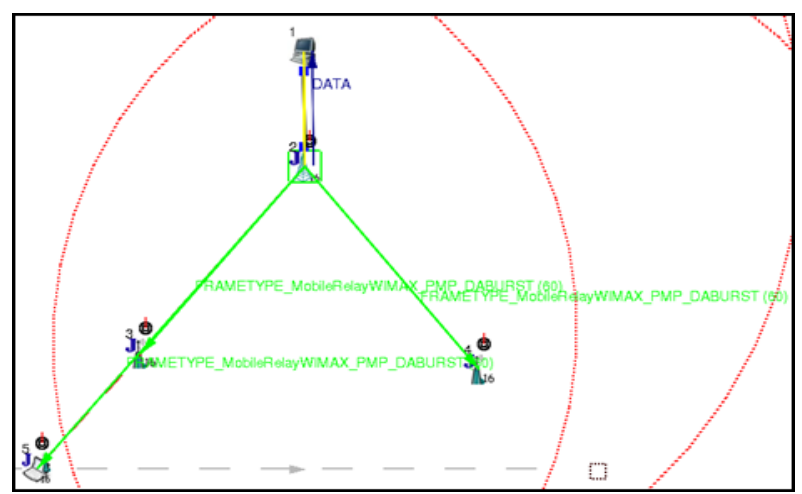

Fig 6: Network topology in NCTUns.

NCTUns is mainly chosen because it supports both transparent and non-transparent modes defined in the IEEE 802.16j. Currently, NCTUns can only support two hops for relaying between BS and MS. Even though the IEEE 802.16j specification can support more than two hops, such a possibility is not supported in NCTUns due to the resulting system complexity. Table 3 presents the IEEE 802.16j parameters used in the simulation.

Table 3. Simulation parameter settings.

\begin{tabular}{l|l}
\hline \hline Parameter & Value \\
\hline Frequency & $2300 \mathrm{MHz}$ \\
Bandwidth (Mbps) & $10 \mathrm{MHz}$ \\
Frame duration & $5 \mathrm{~ms}$ \\
FFT size & 1024 \\
Number of sub-carrier used & 840 \\
DL sub-channel & 30 \\
UL sub-channel & 35 \\
Channel Model & Cost-231 Hata \\
Path Loss Model & Two-ray Ground \\
BS/RS/MS Transmit Power & $43 / 43 / 35 \mathrm{dBm}$ \\
BS/RS/MS Antenna Gain & $15 / 9 / 5 \mathrm{~dB}$ \\
BS/RS/MS Antenna Height & $30 / 20 / 1.5 \mathrm{~m}$ \\
Packet Size (byte) & 1024 \\
Simulation Time & $60 \mathrm{~s}$ \\
\hline \hline
\end{tabular}

In NCTUns, the path selection between MS and BS is influenced by signal-to-noise-ratio (SNR), from which the selection will be based on modulation and coding lookup table.The path decision is based on formula given in Equation (10),

$$
\frac{W_{s}}{W_{r}+W_{p}} \times 100 \%
$$

Note that $W$ is referred to MCS table which defines the relationship between weights, and modulation and coding schemes. Table 2 shows MCS table and path decision based on weightage condition is shown in Fig 7.

$$
\text { If } W_{s}<W_{r}+W_{p}
$$

Direct link between BS-MS is chosen

If $W_{s}>W_{r}+W_{p}$

$R S$ is chosen to be the medium between BS-MS connection

Fig 7: Path decision based on weightage condition.

\subsection{Results and Analysis}

When MS has a data packet to send, it will perform ranging procedure to inform its super-ordinate node by sending signaling message for entering the network. The MS send RNG_REQ message to RS, requesting the RS to provide link. The same procedure happened to RS where RS continue to send RNG_REQ message to BS, requesting the BS to provide link. Then, BS sends its link parameters, i.e. SNR to the corresponding RS and RS sends its link parameters to the corresponding MS. BS decides that corresponding MS access station based on SNR. As depicted in Fig 8, the network entry is accomplishment for RS3, RS4, and MS5 as the BS accept new MS. The relay link mode for both RS, i.e. BS-RS3 and BS-RS4 link is automatically set by BS as Link Mode $=$ Auto $(\mathrm{SNR}=30.958728 \mathrm{~dB}), \quad \mathrm{DIUC}=6 \quad \mathrm{UIUC}=7, \quad$ and Link Mode $=$ Auto $\quad(\mathrm{SNR}=29.798339 \mathrm{~dB}), \quad \mathrm{DIUC}=6 \quad \mathrm{UIUC}=7$, respectively. Meanwhile, the access link mode between BSMS5 is automatically set by BS as Link Mode=Auto $(\mathrm{SNR}=2.418483 \mathrm{~dB}), \mathrm{DIUC}=1 \mathrm{UIUC}=1$.

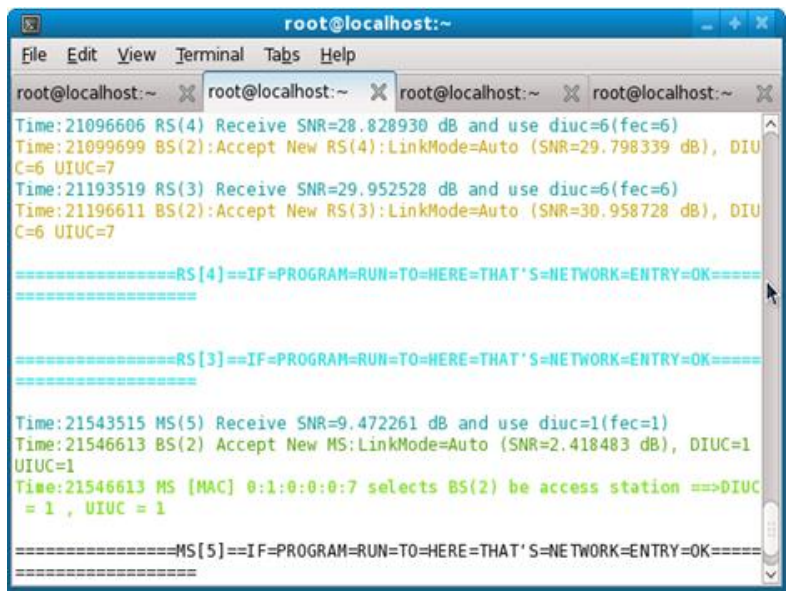

Fig 8: Network entry in NCTUns.

We study the performance of system throughput in terms of Packet Delivery Ratio (PDR) and Packet Loss Ratio (PLR). The performance measures are described as follows:

- Packet Delivery Ratio (PDR): The number of packet received over the number of packet transmitted.

- Packet Loss Ratio (PLR): The number of packet loss over the number of packet transmitted.

As shown in Fig 9, it can be seen that light traffic does not give any big impact to PLR performance since it remains around 0.09 to 0.15 as the traffic load increases. However, heavy traffic which is above $2.0 \mathrm{Mbps}$, give significant impact to PLR performance. Heavy traffic load caused many packets to be generated per second. Since each user had limited available slots, there are several packets need to be queued before transmission. Note that the chance of dropping packets higher in heavy traffic. Thus, increases PLR of one-hop and two-hop communication as the traffic load increases. As observe in Fig 9, the PLR result shows that two-hop communication outperformed one-hop communication as the traffic load increases. 


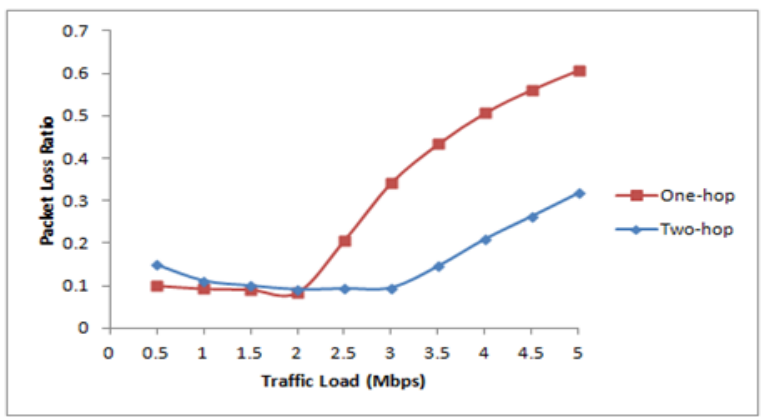

Fig 9: Impact of varying traffic load on the PLR.

The PDR output is shown in Fig 10. It can be seen that light traffic does not give any big impact to PDR performance since it remains around 0.85 to 0.91 as the traffic load increases. However, heavy traffic which is above $2.0 \mathrm{Mbps}$, give significant impact to PDR performance. We can see that as the traffic load increases, the PDR is decrease. Injecting more packets into the network causes the dropping of many of the stored packets. Therefore, the PDR of one-hop and two-hop communication is decreased. However, two-hop communication shows better PDR than one-hop communication as the traffic load increases.

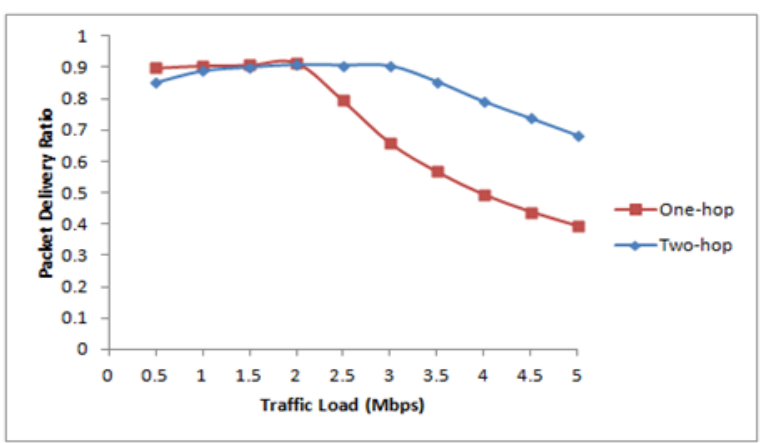

Fig 10: Impact of varying traffic load on the PDR.

Performance output of average end-to-end (ETE) delay is as shown in Fig 11. Average ETE delay defined the total delay of all received packets divided by the number of received packets. The average ETE delay is exponentially decreases as we increase the uplink sustained rate. Note that as we increase the uplink sustained rate, more bits can be delivered per second. Hence, reduce the delay of each packet. However, if

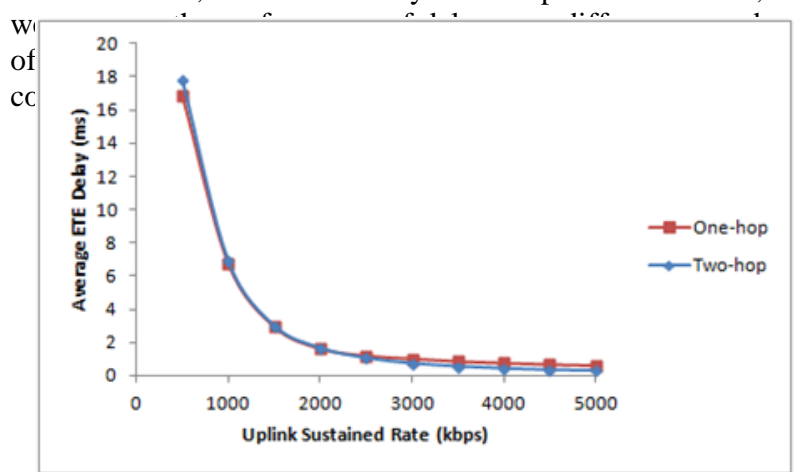

Fig 11: Impact of varying traffic load on the average ETE delay.

The results show that by using multi-hop communication known as relay-based in vehicular network can improve system throughput in terms of PDR, PLR, and average ETE delay.

\section{CONCLUSION AND FUTURE WORK}

In this paper, Dynamic Routing Algorithm (DyRA) with Cross-layer Design (CLD) is proposed for vehicular network. The proposed routing scheme considered metrics at physical and MAC layer in making routing decision at network layer. The preliminary results show that by deploying relay-based in vehicular network can enhance system throughput in terms of PDR, PLR, and average ETE delay. In the future, the performances of proposed routing scheme need to be verified through simulation and analysis.

\section{ACKNOWLEDGMENTS}

The authors would like to thank to Ministry of Science, Technology and Innovation (MOSTI) Malaysia and UTM Research Management Centre (RMC) for the sponsorship and UTM-MIMOS Center of Excellence for the support and advice. Thanks also to all anonymous reviewers for their invaluable comments and the guest editors who handled the review of this paper.

\section{REFERENCES}

[1] Ghassan M. T. Abdalla, Mosa Ali Abu-Rgheff, and Sidi Mohammed Senouci. Current Trends in Vehicular Ad Hoc Networks, Ubiquitous Computing and Communication Journal.IEEE Global Information Infrastructure Symposium. Morocco. July 2007.

[2] Draft Amendment to IEEE Standard for Local and Metropolitan Area Networks - Part 16: Air Interface for Fixed and Mobile Broadband Wireless Access Systems Multihop Relay Specification.IEEE Draft Standard P802.16j/D9. Feb. 2009.

[3] J. Sydir. IEEE 802.16 Broadband Wireless Access Working Group - Harmonized Contribution on 802.16j (Mobile Multihop Relay) Usage Models. July 2006.

[4] Nurul Nazirah M.I.M., N. Satiman, Anis Izzati A.Z., Norsheila Fisla, Sharifah K. Syed Yusof, Sharifah H.S. Ariffin, and Mazlan Abbas. Cross-layer Routing Approach in Highly Dynamic Networks. International Conference on Modeling, Simulation and Applied Optimization (ICMSAO). Pages: 1-5. April 2011.

[5] C. E. Perkins and E. M. Royer. Ad hoc on-demand distance vector routing. IEEE Workshop on Mobile Computing Systems and Applications. New Orleans, LA.Pages: 90-100. 1999.

[6] Tarik Taleb, Ehssan Sakhaee, Abbas Jamalipour, Kazuo Hashimoto, Nei Kato, and Yoshiaki Nemoto. A Stable Routing Protocol to Support ITS Services in VANET Networks. IEEE Transactions on Vehicular Technology. Vol. 56. No. 6. Pages: 3337-3347. Nov. 2007.

[7] S. M. Almajnooni, B. S. Sharif and C. C. Tsimenidis. Mobile Ad Hoc Network for Motorway Transport Applications. IEEE Transactions on Vehicular Technology Conference.Pages: 16-20. April 2007.

[8] Nima Alam, Asghar Tabatabaie Balaie, and Andrew G. Dempster. Dynamic Path Loss Exponent and Distance Estination in Vehicular Network using Doppler Effect and Received Signal Strength. IEEE Transactions on Vehicular Technology Conference. Pages: 1-5. 2010.

[9] N. Brahmi, M. Boussedjra, J. Mouzna, and M. Bayart. Adaptive Movement Aware Routing for Vehicular Ad 
Hoc Networks. International Conference on Wireless Communications and Mobile Computing (IWCMC). Pages: 1310-1315. June 21-24, 2009.

[10] N. Wisitpongphan, O. K. Tonguz, J. S. Parikh,P. Mudalige, F. Bai, and V. Sadekar. Broadcast storm mitigation techniques in vehicular ad hoc networks.IEEE Wireless Communications. Vol. 14. No. 6. Pages: 84-94. 2007.

[11] R. Chen, W. L. Jin, and A. Regan. Broadcasting safety information in vehicular networks: Issues and approaches. Network Magazine of Global Internetworking. Vol. 24. No. 1. Pages: 20-25. 2010.

[12] Nurulashikin Satiman, Rozeha A. Rashid, Norsheila Fisal, N. Nazirah M.I. Ma'arof, Anis I.A. Zamani, S. Kamilah S. Yusof, S. Hafizah S. Ariffin, and Mazlan Abbas. A SNR-based Route Selection Algorithm for WiMAX Mobile Multi-hop Relay Networks. Wireless World Research Forum 2011 (WWRF 26). Doha, Qatar. April 2011.

[13] L. Xiong, L. Libman and G. Mao. On cooperative communication in ad-hoc networks: The case for uncoordinated location-aware retransmission strategies. IEEE Conference on Local Computer Networks (LCN). Zurich, Switzerland.Pages: 554-561.October 20-23, 2009.

[14] R. K. Ganti and M. Haenggi. Analysis of uncoordinated opportunistic two-hop wireless ad hoc systems. IEEE International Symposium on Information Theory.Pages: 1020-1024. 2009.

[15] J. Zhao and G. Cao. VADD: Vehicle-assisted data delivery in vehicular ad hoc networks. IEEE International Conference on Computer Communications. Pages: 1-12. April 2006.

[16] Nawaporn Wisitpongphan, Fan Bai, Priyantha Mudalige, Varsha Sadekar, and Ozan Tonguz. Routing in Sparse Vehicular Ad Hoc Wireless Networks. IEEE Journal on selected areas in Communications. Vol. 25. No. 8. October 2007.

[17] Q. Yang, A. Lim, and P. Agrawal. Connectivity aware routing in vehicular networks. IEEE International Conference on Wireless Communications and Networking Conference (WCNC). Pages: 2218-2223. 2008.

[18] Anis Izzati Ahmad Zamani, Nurul Nazirah Mohd Imam Ma'arof, Farah Buthainah Nor Yusof, Nurulashikin Satiman, Sharifah Kamilah Syed Yusof, and Norsheila Fisal. Cross-layer Relay Selection for Cooperative Relay System in IEEE 802.16j Network. Jurnal Teknologi, 55 (Sains dan Kejuruteraan) Keluaran Khas (1). Penerbit UTM Press, Universiti Teknologi Malaysia. Pages: 255269. May 2011.

[19] N. Satiman, N. Fisal, N.N.M.I. Ma'arof, A.I.A. Zamani, S.K.S. Yusof, and M. Abbas. An Efficient Link Aware Route Selection Algorithm for WiMAX Mobile Multihop Relay Networks. International Journal of Computer Applications. Vol. 27. No. 2. Pages: 48-53. Aug. 2011.

[20] Yu Ge, Su Wen, Yew-Hock Ang and Ying-Chand Liang. Optimal Relay Selection in IEEE 802.16j Multihop Relay Vehicular Networks. IEEE Transactions on Vehicular Technology Conference. Vol. 59. No. 59. Pages: 2198-2206. June 2010.
[21] Ben-Jye Chang, Bo-Jhang Huang and Ying Hsin Liang. Wireless Sensor Network-based Adaptive Vehicle Navigation in Multihop-Relay WiMAX Networks. IEEE Transactions on International Conference on Advanced Information Networking and Applications.Pages: 56-63. 2008.

[22] William Su, Sung-Ju Lee, and Mario Gerla. Mobility Prediction and Routing in Ad Hoc Wireless Networks. International Journal of Network Management. Vol. 11. Issue 1. Pages: 3-30. 2001.

[23] IEEE Standard for Local and Metropolitan Area Networks, Part 16: Air Interface for Fixed and Mobile Broadband Wireless Access System. IEEE Std. 802.16e2005. February 2005.

[24] D.S. Shu'aibu, S.K. Syed Yusof, and N. Fisal. Link Aware Unsolicited Grant Service Packet Scheduling for Mobile WiMAX. International Conference on Modeling, Simulation, and Applied Optimization (ICMSAO). Pages: 1-5. April 2011.

[25] Moritz Killat and Hannas Hartenstein. An Empirical Model for Probability of Packet Reception in Vehicular Ad Hoc Networks. EURASIP Journal on Wireless Communications and Networking. Vol. 2009. Pages: 112. 2009.

[26] Henrik Martikainen, Alexander Sayenko, Oilli Alanen and Vitality Tykhomyroy. Optimal Mac PDU Size in IEEE802.16. IT-NEWS. Pages: 66-71. 2008.

[27] Mahesh Kumar Yadav, Ram Kishan Khola, and Deepak Dembla. Modeling, Analysis \& Implementation of Improved AODV Routing Protocol in MANETs, International Journal of Computer Applications.Vol. 41.No. 21. Pages: 37-42. March 2012.

[28] Brij Bihari Dubey, Naveen Chauhan, and Prashant Kumar. A Survey on Data Dissemination Technique used in VANETs. International Journal of Computer Applications. Vol. 10, No. 7.Pages: 5-10. Nov. 2010.

[29] Abey Abraham and Jebapriya S. Routing Strategies in Delay Tolerant Networks: A Survey. International Journal of Computer Applications. Vol. 42. No. 19. Pages: 44.48. March 2012

[30] G. Hunag, Y. Wang, J. Coon. Performance of Multihop Decode-and-Forward and Amplify-and-Forward Relay Networks with Channel Estimation. Pacific Rim Conference on Communication, Computers and Signal Processing. Pages: 352-357. August 2011.

[31] A. Bou Saleh, S. Redana, B. Raaf, T. Riihonen, J. Hamalainen, R. Wichman. Performance of Amplify-andForward and Decode-and-Forward Relays in LTEAdvanced. $70^{\text {th }}$ Vehicular Technology Conference. Pages: 1-5. September 2009.

[32] S. Amara, H. Boujemaa, N. Hamidi. SEP of Cooperative Systems using Amplify and Forward or Decode and Forward Relay over Nakagami-m Fading Channels. International Conference on Signals, Circuits and Systems. Pages: 1-5. November 2009. 